\title{
FUNCTIONAL OUTCOMES OF STROKE PATIENTS ADMITTED TO A TERTIARY HOSPITAL IN THE WESTERN CAPE, SOUTH AFRICA
}

\begin{abstract}
Individuals with stroke often experience functional limitations such as the inability to walk independently. There are several factors that are associated with functional outcome post stroke. The aim of the study was therefore to determine functional outcomes and factors influencing functional outcomes of stroke patients admitted to a South African tertiary hospital. A longitudinal design was used to obtain the data. A convenient sampling method was used to recruit 100 participants. Data was collected on admission, discharge and two months post stroke, using the National Institute of Health Stroke Scale, Barthel Index and a socio-demographic and medical profile data sheet. The data was analyzed using

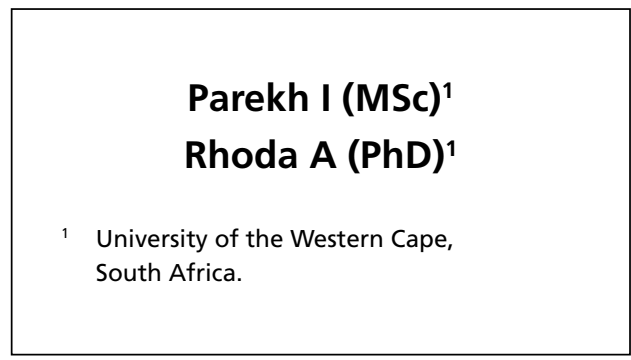
SPSS version 18 and SAS version 9. Tobit analysis was used to determine the factors influencing functional outcomes at discharge and two months post stroke. The necessary ethical clearance was obtained prior to commencement of the study. Stair climbing, mobility and transfers were the functional activities that the majority of the participants could not perform independently. Age and severity of stroke significantly influenced the functional outcomes at discharge and two months post stroke. Factors such as age and stroke severity should therefore be considered when planning rehabilitation interventions for stroke patients in this setting.
\end{abstract}

KEY WORDS: STROKE, FUNCTIONAL OUTCOMES, SEVERITY OF STROKE, SOUTH AFRICA

\section{BACKGROUND}

Stroke is a common global health care problem that is serious and disabling (Warlow et al 2008). If viewed within the International Classification of Functional Disability and Health, the impact of stroke can be characterised by a number of impairments, activity limitations and participations restrictions that are experienced post stroke. The most common impairments identified are motor and sensory function, abnormal muscle tone, cognitive and perceptual limitations, speech impairments, depression, urinary incontinence and dysphagia (Lawrence et al 2001). Patients who have

\section{Correspondence Author:}

Ishita Parekh

103 Jabral Court,

22 Main Road, Rosebank 7700

Cape Town

Republic of South Africa

Email: iparekh87@gmail.com experienced a stroke have limitations in performing self-care, household and community activities (Rhoda 2011).

Various studies identified different factors associated with outcome post stroke (Meijer et al 2003; Gialanella et al 2012). Disability on admission, swallowing problems, age, severe paresis and urinary incontinence were found to be predictors of functional outcome post stroke (Meijer et al 2003). Ability to perform basic activities of daily living is also an important predictor of stroke outcome (Gialanella et al 2012). Furthermore, factors relating to the structure of rehabilitation have been found to affect the outcome post stroke. Strong evidence exists for the rehabilitation of patients with stroke in specialized stroke units. Organized inpatient stroke units are effective in improving short-term survival, functional ability, independence and the need for institutionalization (Stroke Unit trialists' Collaboration 2007). In
South Africa, the stroke unit model of care has not been widely implemented despite robust evidence of its efficacy (Bryer et al 2010). Further research is needed regarding the management of stroke patients in stroke units in South Africa. In the recent years information relating to outcomes of stroke patients admitted to other levels of healthcare in the Western Cape have been published (Rhoda et al 2011; Rouillard et al 2012), but no published literature is available regarding the outcomes of patients discharged from a tertiary hospital which has a stroke unit in the Western Cape. Milinaviciene et al (2007) stated that for effective utilization of rehabilitation resources, identifying various factors affecting functional outcome is important. The aim of the current study was therefore to determine the functional outcomes and factors affecting these outcomes of stroke patients admitted to a tertiary acute care hospital in the Western Cape, South Africa. 


\section{METHODOLOGY}

The study was conducted on 100 stroke patients consecutively admitted at Groote Schuur Tertiary hospital, Cape Town for a 7-month period that occurred from 1 October 2010 to 30 April 2011. A descriptive, longitudinal, quantitative design was used. A convenient nonprobability sampling method was used. The inclusion criteria were first ever occurrence of stroke, onset $\leq 4$ weeks prior to admission and provision of written informed consent either by the patient or the caregiver. Patients were excluded from the study if they had additional neurological conditions such as spinal cord injury, head injury, dementia or history of seizures prior to stroke. Patients with speech problems were included in the study.

A self-developed, socio-demographic and medical profile data sheet was used to capture the socio-demographic and medical profile data from the medical records of the patients. Data collected on admission included age, gender, level of education, employment status, documented risk factors for stroke, type of stroke, side of lesion and side of impairment. The variables captured from participants' folders on discharge were frequency and type of rehabilitation and length of hospital stay. The face and content validity of the data sheet was determined by it being reviewed by experts in the field of stroke management. The National Institutes of Health Stroke Scale (NIHSS) (Brott et al 1989) was used to determine the severity of stroke on admission and discharge. The scale is completed by direct observation as opposed to self-report. The NIHSS includes the following 15 items; cognitive impairment, gaze deviation, hemianopia, facial palsy, weakness of lower and upper limbs, ataxia of lower and upper limbs, sensory loss, dysarthria, aphasia and inattention. The scale is scored from $0-42$, with a higher score indicating a higher level of neurological impairment or stroke severity. The intraclass correlation coefficient for the NIHSS was documented as between 0.93 to 0.95 (Godstein and Samsa 1997). The scale was found to be valid and shows concurrent validity with the Barthel Index (BI). Good agreement has been documented between correlation coefficients when comparing the NIHSS with the BI, the Rankin Scale, and the Glasgow Outcome (Lyden et al 1999).

The Barthel Index (BI) (Mahoney and Barthel 1965) was used to capture data relating to the functional ability of the participants. The aim of the scale is to measure the level of independence in the basic activities of daily living. The scores range from $0-100$ with higher scores indicated higher level of functioning (Finch et al 2002). Information needed to complete the BI could be obtained from the most reliable source, which could be the health care worker or the carer (Finch et al 2002). In the current study the researcher consulted with the nursing staff and the patients caregiver when completing the BI. An excellent inter-rater reliability of $r=0.489 \quad(p<0.001)$ has been found for this tool (Rollnik 2011). The reliability of telephonic interview was also found excellent (95\% CI, 0.85-0.94) (Pietra et al 2011).

The researcher visited the medical ward and stroke unit on a daily basis to identify patients who could be included into the study. Patients who were eligible to be included were invited to participate in the study with the aim of the study being explained to them. Written informed consent was obtained from relevant patients or proxies when the patient was unable to respond. Data were collected on admission, discharge and 2 months post stroke. After completing the socio-demographic and medical profile data sheet, the researcher completed the NIHSS and BI as above. These instruments were completed in privacy at a time that was convenient for the patient. The same instruments were completed with each participant at discharge with a window period of two days. At 2 months post discharge, telephonic interviews for the BI were conducted. The caregivers of patients who were unable to be interviewed via the telephone were interviewed as proxy respondents.

\section{DATA ANALYSIS}

The data was captured using the Statistical Package for the Social Sciences (SPSS) version 19 while analyzed using SPSS version 19 and Statistical Analysis System (SAS) version 9. Descriptive statistics such as mean, median, mode and standard deviation were calculated using SPSS. Due to the type and distribution of the data Spearman's correlation was used to determine the correlation between the outcome variable which was the Barthel score at discharge and two months post stroke and each potential predictor variable (age, frequency of rehabilitation, severity at admission and functional level at admission). Tobit analysis was used to determine factors influencing the outcome variable at discharge and 2 months post stroke. The Tobit model also called the censored regression model (Yang and Douglas 2010) was used to address the ceiling and floor effects noted in the outcome. The significance level for inclusion in the final regression was $\mathrm{p}<0.0001$. The data of the patients who died, at discharge $(n=6)$ and 2 months $(n=6)$ were included in the analysis and BI score, as zero was used.

\section{Ethics}

Ethical clearance to conduct the study was obtained from the Senate Higher Degrees Committee of the University of the Western Cape and the Senate research grants and study leave committee of UWC. Permission was also obtained from the Medical Superintendent of Groote Schuur Hospital and the Chairperson of Health Science Faculty Research Ethics Committee at University of Cape Town. The aims, objectives and nature of the study were explained to all the participants. If they agreed to participate in the study then written informed consent was obtained from them or a primary caregiver. The participants were informed of their right to withdraw and that confidentiality and anonymity would be maintained during the collection, analysis and publication of the data.

\section{RESULTS}

\section{Socio-demographic and medical factors}

A total of 100 participants were recruited to be part of the study. The mean age of the participants was $59.83(\mathrm{SD}=15.71)$ 
years with $51 \%$ females and $49 \%$ males. Hypertension was found to be the most common risk factors $79 \%$, with smoking being the second most common risk factor $49 \%$ among the participants. Ischemic stroke was found in $89 \%$ while hemorrhagic stroke was in $10 \%$. Six percent died before discharge and $6 \%$ after discharge but before 2 months follow-up assessment period.

\section{Rehabilitation process factors}

The number of patients that received Physiotherapy (75\%) and Occupational Therapy (79\%) was nearly similar. The mean length of stay of patients was $10.4(\mathrm{SD}=5.80)$ days. More than half of the patients $53.1 \%$ were referred for follow-up to inpatient rehabilitation centers and $46.8 \%$ were referred for follow-up to outpatient rehabilitation.

\section{Severity of stroke}

The mean of NIHSS was 12.49 and 8.76 on admission and discharge respectively. Missing data of one participant and demise of six participants reduced the total number of patients as ninetythree for the discharge NIHSS findings. On admission $21 \%$ of the patients had a severe stroke, which reduced at discharge and remained severe for $9.6 \%$ of the participants excluding those who died. The number of participants with minor stroke increased from $22 \%$ on admission to $30.2 \%$ at discharge. The majority of participants experienced a moderate stroke with $52 \%$ on admission and $44 \%$ at discharge.

\section{Functional outcome}

The mean and standard deviation (SD) of the BI scores was greater at 2 months 55(40.3) than on discharge 43(35) and admission 28(29).

Figure 1 illustrates the BI scores of the participants at the three assessment points; admission, discharge and 2 months post stroke. The majority (77\%) of participants were totally dependent on others to perform functional activities on admission. Functional outcome increased over time but $43.9 \%$ of participants were still dependent at 2 months post stroke.

Table 1 presents the number of participants who were independent on

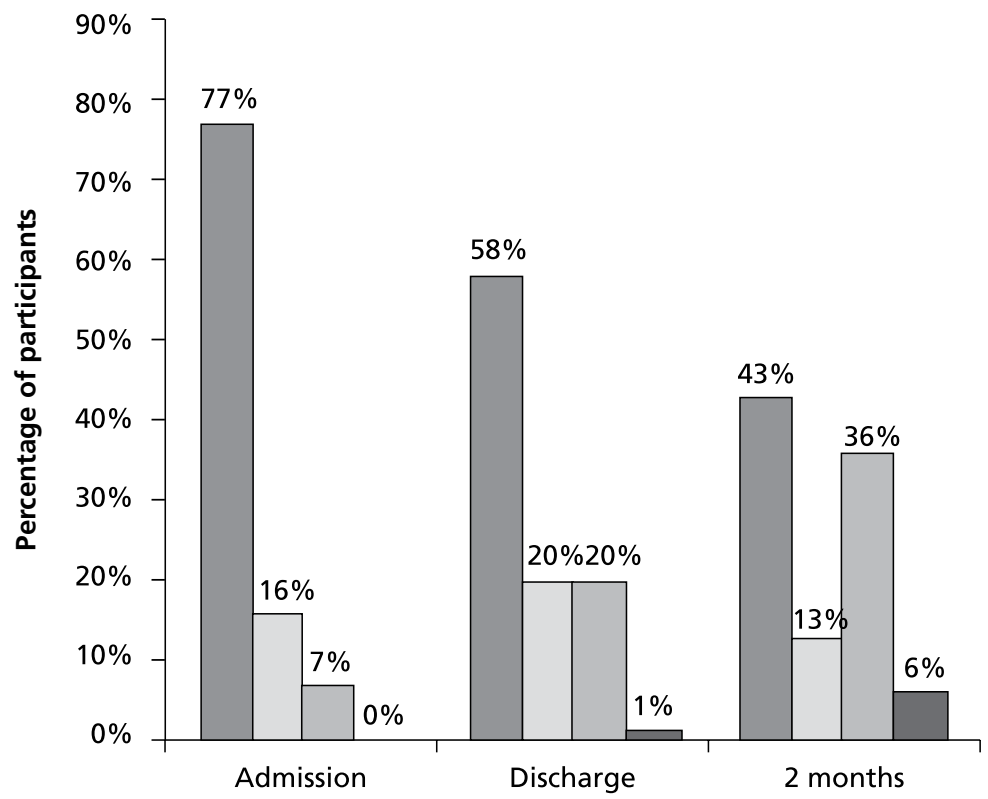

Functional outcomes of participants

Dependent (0-55)

Moderate assistance (60-80)

Minimal assistance (85-95)

Independent (95-100)

Figure 1: Functional outcome as measured by $\mathrm{BI}$ at different assessment periods

Table 1: Descriptive statistics of BI

\begin{tabular}{|l|l|l|l|}
\hline Individual items & $\begin{array}{l}\text { Admission }(\mathrm{n}=100) \\
\text { Number }(\%)\end{array}$ & $\begin{array}{l}\text { Discharge }(\mathrm{n}=99) \\
\text { Number }(\%)\end{array}$ & $\begin{array}{l}\text { 2 months }(\mathrm{n}=66) \\
\text { Number }(\%)\end{array}$ \\
\hline Feeding & $61(61)$ & $77(77.7)$ & $51(77.2)$ \\
\hline Bathing & $57(57)$ & $69(69.6)$ & $48(72.7)$ \\
\hline Grooming & $53(53)$ & $65(65.6)$ & $46(69.6)$ \\
\hline Dressing & $42(42)$ & $57(57.5)$ & $43(65.1)$ \\
\hline Bowel & $33(33)$ & $48(48.4)$ & $41(62.1)$ \\
\hline Bladder & $25(25)$ & $42(42.4)$ & $39(59.0)$ \\
\hline Toilet & $23(23)$ & $39(39.3)$ & $38(57.5)$ \\
\hline Transfer & $13(13)$ & $29(29.2)$ & $35(53.0)$ \\
\hline Mobility & $2(2)$ & $9(9.0)$ & $26(39.3)$ \\
\hline Stairs & 0 & $1(1.0)$ & $4(6.0)$ \\
\hline
\end{tabular}


different items of the BI at different assessment periods. The activities that the majority of participants needed assistance with at all three assessment periods, was stair climbing followed by mobility and transfers.

\section{Correlation between outcome variable and potential variable}

The results of the Spearman's correlation indicated that the total number of rehabilitation sessions was not significantly correlated with functional outcome at discharge $(p=0.0842)$. At discharge, age $(p<0.0001)$, severity of stroke on admission $(\mathrm{p}<0.0001)$, and functional levels on admission $(p<0.0001)$, correlated significantly with functional outcome while at 2 months post stroke, age and severity of stroke on discharge correlated significantly with functional outcome. These variables were therefore included appropriately in the regression analysis to determine the factors influencing functional outcome at discharge and 2 months post stroke.

\section{Factors influencing functional out- come at discharge}

The factors that were identified as those influencing functional outcome were age, stroke severity and functional level on admission. The analysis of maximum likelihood parameter estimate showed that the coefficient for age was -0.50 . For each year increase in age, the estimated mean would decrease by 0.50 . For every ten year increase in age, the discharge functional score would decrease by 5-points. The coefficient for functional level at admission was 0.55 . For 1-point increase in admission functional score, the discharge functional score would increase by 0.5 . The coefficient for severity at admission was 1.75. A 1-point increase in severity would correspond to 1.75-point decrease in discharge functional score.

\section{Factors influencing functional out- come at 2 months}

The analysis of maximum likelihood parameter estimated that age $(\mathrm{p}=0.0143)$ and severity at admission $(\mathrm{p}<0.0001)$ were both significant together for functional outcome at 2 months. The coefficient for age was -0.61 . Each one year increase in age would decrease the functional score by 0.61 . Each ten year increase in age would decrease it by 6-points. The coefficient for severity at admission was -3.50 meaning each 1 -point increase in severity at admission corresponded to an estimated 3.50 decrease in functional outcome at two months.

\section{DISCUSSION}

\section{Participant characteristics}

A total of 100 participants were recruited to be part of the study. The mean age of the participants, $59.83(\mathrm{SD}=15.71)$ years, was younger than stroke patients admitted to inpatient facilities in developed countries, which was reported as 75 years (Lee et al 2011). The mean age of the participants were however similar to stroke patients admitted to inpatient rehabilitation centers in the Western Cape. Rouillard (2006) and Bryer (2009) reported the age of stroke patients admitted to inpatient facilities in the Western Cape as 51.3 years and 59 years respectively. The patients were however slightly younger than the stroke patients managed at Community Health Centres (CHC) in the Western Cape where a mean age of $61(\mathrm{SD}=10.5)$ years was reported (Rhoda et al 2011). It is not clear why the mean age of the patients is much lower in sub-Saharan African countries than other countries. From the results of these local studies it appears that stroke patients being admitted for inpatient management are younger than those managed at outpatient facilities. The limited resources for rehabilitation in developing countries could be the reason for this as younger patients are admitted for intense rehabilitation as they have better outcomes than older ones (Varona 2011).

As found in previous studies (Rhoda et al 2003; Rouillard et al 2012) conducted in the Western Cape, hypertension was the most prevalent risk factor for stroke. It was found that the knowledge of stroke risk factors was inadequate among the South African population (Bogoshi et al 2003). This lack of knowledge about the risk of stroke could be resulting in stroke being a cause of morbidity and mortality in this country.
Functional outcome of stroke on admission, discharge and 2 months The functional outcomes of the individuals were determined using the BI. The participants admitted to the tertiary hospital needed assistance with mobility, stair climbing and transfers. With exception of stair climbing these were different to the activities that patients in the previous studies conducted in the Western Cape needed assistance with. Participants in the studies conducted by Rhoda et al (2011) and Rouillard et al (2012) were dependent in activities such as feeding, washing and stair climbing. In the present study the majority of participants were dependent in daily activities, scoring between 0 and 55 on $\mathrm{BI}$ at the time of admission (Granger et al 1979). It should however be noted that in the acute stage, the dependent state does not always mean inability to perform activities of daily living it could be linked medical directive. At discharge the majority of participants were still however dependent. The reason for this could be the short length of stay (mean=10.4 days) which patients admitted to tertiary institutions experience. At 2 months, a significant improvement was however noted for individual items on BI scores. The majority $(43.9 \%)$ of participants still however needed maximum assistance. There is also a concern that early benefits achieved by acute stroke unit rehabilitation could be eroded by a lack of adequate rehabilitation after discharge (De Villers et al 2009). The study of Wang et al (2011) suggests that earlier transfer to an inpatient rehabilitation hospital leads to improved functional outcome. However, certain factors such as age, initial medical condition and functional status contribute to functional improvement.

\section{Factors affecting functional out- comes at discharge and 2 months post stroke}

Increasing age was found to be significantly associated with poor functional outcome $(p<0.0001)$ at discharge and two months. This finding could be as a result of additional disabilities and comorbidities, which are associated with increased age (Nichols-Larsen et 
al 2005). As confirmed by the findings of this study severity of stroke was an important factor, which could be used to determine the outcome post stroke This is an important finding in the countries like South Africa where the resources for stroke rehabilitation is limited which could consider stroke severity as an admission criteria. It should however be noted that if structured interdisciplinary stroke units are available these patients with severe stroke have improved outcomes.

Functional level on admission predicted functional level at discharge and 2 months post stroke. This finding supports that of a previous study conducted by Tilling et al (2001). The level of functional ability at admission could therefore be used to predict functional level at discharge and be used to assist with discharge planning.

\section{CONCLUSION}

It can therefore be concluded from the results of the study that age, severity of stroke and functional level at admission are the factors that affect functional outcome at discharge and 2 months post stroke of patients admitted to a tertiary hospital in the Western Cape, South Africa. Therefore, the rehabilitation staff should consider these factors while planning rehabilitation interventions and referral to rehabilitation facilities for patients admitted to this setting. In addition rehabilitation of elderly patients could focus more on task oriented activities and compensation rather than on recovery of neurological status.

\section{REFERENCES}

Bogoshi GM, Stewart A, Hale L, Fritz V 2003 Knowledge of stroke risk factors amongst black diabetic, hypertensive and stroke patients. South African Journal of Physiotherapy 59(4):25-30

Brott HP, Adams CP, Olinger JR, Marler WG, Barsan J, Biller J, Spilker R, Holleran R, Eberle V, Hertzberg M, Rorick CJ, Moomaw MW 1989 Measurements of acute cerebral infarction: a clinical examination scale. stroke, 20:964-970

Bryer A 2009 The need for a community-based model for stroke care in South Africa. South African Medical Journal Cape Town 99(8)
Bryer A, Connor M, Haug P, Cheyip B, Staub H, Tipping B, Duim W, Pinkney-Atkinson V 2010 South African guideline for management of ischaemic stroke and transient ischaemic attack a guideline from the South African Stroke Society (SASS) and the SASS Writing Committee. South African Medical Journal 100:747-78

De Villiers L, Kalula S, Burch V 2009 Does multidisciplinary stroke care improve outcome in a secondary level hospital in South Africa? International Journal of Stroke 2:89-93

Finch E, Brooks D, Stratford PW, Mayo NE 2002. Physical rehabilitation outcome measures: A guide to enhanced clinical decision-making Baltimore (MD): Lippincott Williams \& Wilkins

Gialanella B, Santoro R, Ferlucci C 2012 Predicting the outcome after stroke: the role of basic activities of daily living. European Journal of Physical and Rehabilitation Medicine (ahead of print)

Goldstein LB, Samsa GP 1997 Reliability of the National Institutes of Health Stroke Scale. Extension to non-neurologists in the context of a clinical trial Stroke 28(2):307-310

Granger C, Dewis L, Peters N, Sherwood C, Barret J 1979 Stroke Rehabilitation: analysis of repeated Barthel Index Measures. Archives of Physical Medicine and Rehabilitation 60:14-17

Lawrence E, Coshall C, Dundas R, Stewart J, Rudd AG, Howard R, Wolf CD 2001 Estimates of the prevalence of acute stroke impairments and disability in a multiethnic population. Stroke 32(6):1279-84

Lyden P, Mei L, Jackson C, Marler J, Kothari R, Brott T 1999 Underlying structure of the National Institutes of Health Stroke Scale: Results of a factor analysis. Stroke, 30:2347-2354

Lee S, Shafe A, Cowie M 2011 UK Stroke incidence, mortality and cardiovascular risk management 1999-2008: time-trend analysis from the General Practice Research Database. British Medical Journal Open 1(2):e000269

Mahoney FI, Barthel DW 1965 Functional evaluation: the Barthel Index. Maryland state medical journal Feb;14:61-65

Meijer R, Ihnenfeldt DS, de Groot IJ, van Limbeek J, Vermeulen M, de Haan RJ 2003 Prognostic factors for ambulation and activities of daily living in the subacute phase after stroke. A systematic review of the literature. Clinical Rehabilitation Mar;17(2):119-129

Milinaviciene E, Rastenyte D, Krisciūnas A 2007 Factors influencing outcomes in patients with stroke. Medicina (Kaunas) 43(4):269-77

Nichols-Larsen DS, Clark PC, Zeringue A, Greenspan A, Blanton S 2005 Factors influencing stroke survivors' quality of life during subacute recovery. Stroke 36(7):1480-1484
Pietra GLD, Katia S, Elodie O, Monica R, Francesco M, Maurizio AL 2011 Validity and Reliability of the Barthel Index Administered by Telephone. Stroke 42:2077-2079

Rhoda A, Hendry J 2003 Profile of stroke patients treated at a Community-Based rehabilitation Centre in a Cape Town Health District. South African Journal of Physiotherapy 59(4):20-24

Rhoda A, Mpofu R, De Weerdt W 2011 Activity limitations of patients with stroke attending out-patient facilities in the Western Cape, South Africa. South African Journal of Physiotherapy 67(2):16-22

Rollnik JD 2011 The early rehabilitation Barthel Index (ERBI). Die Rehabilitation 50(6):408-411

Rouillard S 2006 Pattern of recovery and outcome after stroke in patients accessing a Western Cape rehabilitation facility. Cape Town

Rouillard S, Willy De Weerdt W, De Wit L, Jelsma J 2012 Functioning at 6 months post stroke following discharge from inpatient rehabilitation. South African Medical Journal 102(6):545-48

Stroke Unit Trialists' Collaboration 2007. Organised inpatient (stroke unit) care for stroke. Cochrane Database of Systematic Reviews, Issue 4. Art. No.: CD000197

Varona J 2011 Long-term prognosis of ischemic stroke in young adults. Stroke Research and Treatment. Vol. 2011,Article ID 879817, 5 pages, doi: $10.4061 / 2011 / 879817$

Warlow CP, van Gijn J, Dennis MS, Wardlaw J, Bamford J, Hankey G, Sandercock P, Rinkel P, Langhorne P, Sudlow C, Rothwell P 2008 Stroke: practical management (3rd edn.) Blackwell Publishing, Oxford

Wang H, Camicia M, Terdiman J, Hung Y, Sandel E 2011 Time to Inpatient Rehabilitation Hospital Admission and Functional Outcomes of Stroke Patients. Physical Medicine and Rehabilitation 3:296-304

Yang Y, Simpson DG 2010 Conditional decomposition diagnostics for regression analysis of zero-inflated and left-censored data. Statistical Methods in Medical Research 0(0):1-16 